\section{OP002/\#213 IS CERVICAL EXCISION BEFORE RADICAL HYSTERECTOMY ASSOCIATED WITH BETTER ONCOLOGIC OUTCOMES FOR PATIENTS WITH EARLY STAGE CERVICAL CARCINOMA ?}

D Nasioudis*, E Ko, A Haggerty, L Cory, R Giuntoli li, S Kim, N Latif. University of Pennsylvania, Division of Gynecologic Oncology, Philadelphia, USA

10.1136/ijgc-2021-IGCS.19

Objectives Investigate the prognostic significance of prior cervical excision procedure (EXC) for patients with early-stage cervical carcinoma undergoing radical hysterectomy.

Methods Patients with FIGO 2009 stage IB1 cervical carcinoma, no history of another tumor who underwent between 2004-2015 a radical hysterectomy, with $\geq 10$ lymph nodes (LNs) removed, known mode of surgery and at least 1 month of follow-up were drawn from the National Cancer Database. Patients who did and did not undergo EXC (within 3 months from radical hysterectomy) were identified. Overall survival (OS) was compared with the log-rank test while a Cox model was constructed to control confounders.

Results A total of 3159 patients were identified; 37.1\% $(n=1171)$ had EXC while 55.9\% $(n=1766)$ underwent minimally-invasive surgery (MIS). Patients who had EXC were less likely to have laparotomy $(39.5 \%$ vs $46.8 \%, \quad \mathrm{p}<0.001)$, lymph-vascular invasion (LVSI, 29.2\% vs $34.9 \%, \mathrm{p}=0.014$ ), positive LNs $(6.7 \%$ vs $12.7 \%, \mathrm{p}<0.001)$, and tumors $>2 \mathrm{~cm}$ $(25.7 \%$ vs $56 \%, \mathrm{p}<0.001)$. For patients with tumors $\leq 2 \mathrm{~cm}$ $(\mathrm{p}=0.008)$ and $>2 \mathrm{~cm}(\mathrm{p}=0.004)$, EXC was associated with better OS. After controlling for mode of surgery, tumor size, histology, LN status, LVSI, age, insurance status and comorbidities, patients who had EXC had better OS (HR: 0.45, 95\% CI: $0.30,0.66)$ compared to those who did not. After controlling for confounders there was no OS difference between laparotomy with EXC, and MIS with EXC (HR: 1.37, 95\% CI: $0.66,2.82)$.

Conclusions Cervical excision before radical hysterectomy may be associated with a survival benefit for patients with stage IB cervical cancer.

\section{OP003/\#32 PREFERENCES AND EXPERIENCES REGARDING THE USE OF THE SELF-SAMPLING DEVICE IN THE HRHPV SCREENING FOR CERVICAL CANCER}

${ }^{1} \mathrm{M}$ Dieleman*, ${ }^{2} \mathrm{~J}$ De Waard, ${ }^{2} \mathrm{~B}$ Wisman, ${ }^{2} \mathrm{E}$ Schuuring, ${ }^{3} \mathrm{M}$ Esajas, ${ }^{1} \mathrm{~K}$ Vermeulen, ${ }^{1} \mathrm{~T}$ De Bock. 'University Medical Centre Groningen, Epidemiology, Groningen, Netherlands; ${ }^{2}$ University Medical Centre Groningen, Pathology, Groningen, Netherlands; ${ }^{3}$ University Medical Centre Groningen, Gynaecology and Obstetrics, Groningen, Netherlands

\subsection{6/ijgc-2021-IGCS.20}

Objectives To improve participation in the cervical cancer screening, a self-sampling device (SSD) has been introduced in 2017 into the Dutch population-based screening programme (PBS). The aim of this study was to gather potential preferences and experiences that might influence a woman's decision to use the SSD in the Dutch PBS.

Methods A systematic literature research was performed in the PubMed database. Studies that assessed preferences and experiences of women regarding the SSD were included and preferences and experiences were extracted. In addition, the list of potential preferences and experiences was extended based on semi-structured interviews with SSD-users as well as not-SSD-users who recently participated in the Dutch PBS.

Results Seventy-six studies were included in the literature research and sixteen interviews were performed. Frequently mentioned preferences and experiences for (not) using the SSD were: practicality, comfort, fear of not performing the SSD procedure correctly, and doubts on whether the results of the high-risk human papillomavirus (hrHPV) test will be reliable. New preferences and experiences elicited in the interviews were: accessibility, not being aware the SSD was an option and the inconvenience that after an hrHPV-positive test result of the SSD, an additional smear test at the GP is necessary.

Conclusions Several preferences and experiences play a role in the choice whether or not to use the SSD. Based on the currently found preferences and experiences, an app will be developed in order to assess which of these are the most important for women participating in the Dutch PBS.

\section{OP004/\#35 BYL719 (ALPELISIB) FOR THE TREATMENT OF PIK3CA-MUTATED, RECURRENT/ADVANCED CERVICAL CANCER}

${ }^{1} \mathrm{G}$ Bogani*, ${ }^{2} \mathrm{M}$ Bini, ${ }^{1} \mathrm{D}$ Ronzulli, ${ }^{1} \mathrm{~F}$ Raspagliesi. ${ }^{1}$ Fondazione IRCCS Istituto Nazionale dei Tumori di Milano, Gynecologic Oncology, Milano, Italy; ${ }^{2}$ Fondazione IRCCS Istituto Nazionale dei Tumori di Milano, Oncology, Milano, Italy

\subsection{6/ijgc-2021-IGCS.21}

Objectives Advanced/recurrent cervical cancer has limited therapeutic options, with a median progression-free survival (PFS) after the failure of systemic treatments ranging between 3.5 and 4.5 months. Here, we reported our preliminary experience in the use of BUL719 (alpelisib) in advanced/recurrent cervical cancer after failure of at least 2 lines of treatment.

Methods The Istituto Nazionale dei Tumori di Milano (Italy) approved this prospective investigation. From 04/01/2020 to 09/01/2020, 17 consecutive patients with recurrent cervical cancer underwent NGS to assess the presence of PIK3CA mutation/alteration.

Results Six patients were included in the study. All patients had been treated with at least 2 previous lines of systemic treatment: 3 patients received $>2$ prior lines of treatment in the recurrent or metastatic setting; $60 \%$ had received prior bevacizumab in combination with chemotherapy. All patients started alpelisib at the daily dosage of $300 \mathrm{mg}$. Investigatorassessed confirmed objective response rate (ORR) was 33\%. The disease control rate (DCR) was $100 \%$. According to the RECIST 1.1, two patients had a partial response (PR), and four patients had stable disease (SD). No complete response was observed. The mean duration of response (DOR) was 6.6 (SD 3.75) months; four patients had PR lasting for $>6$ months. One patient stopped the treatment at 0.82 months due to the onset of a grade 2 adverse event (AE) (skin rash). Grade 3 treatment-related AEs included: lymphoedema $(n=1$, $20 \%)$ and rash $(n=1,20 \%)$. No treatment-related grade $4-5$ AEs occurred.

Conclusions Further trials are needed to assess the safety and effectiveness of alpelisib in PIK3CA-mutated recurrent/ advanced cervical cancer. 